\title{
Diseño y desarrollo de una aplicación móvil accesible de navegación individual y localización para personas de la tercera edad con discapacidad visual
}

\author{
Etelvina Archundia, Carmen Cerón, Patricia Cervantes, Fernando Rodríguez \\ Benemérita Universidad Autónoma de Puebla, \\ Facultad de Ciencias de la Computación, \\ México \\ \{etelvina, mceron\}@cs.buap.mx, \\ \{cervantes.patty, ferrgzt\}@gmail.com
}

\begin{abstract}
Resumen. El propósito del artículo se centra en el Diseño y desarrollo de una aplicación móvil para apoyar la navegación individual y localización de personas de la tercera edad con discapacidad visual. Para el desarrollo de la aplicación móvil, se utilizó la metodología Extreme Programación, el Diseño Universal, y las tecnologías integradas de WebService, PHP, MYSQL, Java y Apache. La aplicación permite la ubicación del sujeto y compartirla entre distintos usuarios, con la finalidad de brindar seguridad y facilitar una mayor inclusión del uso de la tecnología a esta población. Finalmente se presentan los resultados obtenidos de una prueba piloto mediante la experiencia de los usuarios en distintos escenarios.
\end{abstract}

Palabras clave: Discapacidad visual, usabilidad, apps, adulto mayor.

\section{Design and Development of an Accessible Mobile App of Individual Navigation and Localization for Elder People with Visual Disability}

\begin{abstract}
The purpose of this article is focused on the design and development of an accessible mobile app of individual navigation and location for elder people with visual disability. To develop the mobile application, it was used as methodology Extreme Programming, el Universal Design, integrated technologies of WebService, PHP, MYSQL, Java and Apache. The app allows to know the man's location and share it within many users providing security and facilitate a better inclusion of the use of technology to this kind of population. Finally the article shows results obtained from a pilot test through the user's experience in different scenarios.
\end{abstract}

Keywords: Visual disability, usability, apps, elder people. 


\section{Introducción}

Las tecnologías de la información y la comunicación (TIC) proporcionan, en principio, los mismos beneficios a las personas mayores que al resto de la población: acceso a la comunicación, información, ocio, servicios que desde internet facilitan la vida diaria. Sin embargo se requieren aplicaciones accesibles para las personas mayores que permitan manejarlas con la menor carga cognitiva e interfaces fáciles de manejar. Además se han identificado en las personas de la tercera edad alguna discapacidad auditiva o visual, disminuyendo la agudeza visual, auditiva y la destreza manual, esto conlleva a ser necesario desarrollar herramientas o artefactos tecnológicos para apoyarlos en su entorno de trabajo, familiar y social [1].

El actual envejecimiento de la población es un acontecimiento demográfico que nunca antes se había vivido en la historia de la humanidad dado el descenso de la natalidad y la prolongación de la vida media de las personas. Los índices de envejecimiento siguen creciendo desde hace años de forma desmesurada lo que conlleva a importantes repercusiones a nivel social y sanitario [2]. Por otro lado, vivimos en una sociedad donde el volumen de nuevos conocimientos e información crece de forma acelerada y en progresión geométrica en todos los sectores de la vida social. A este hecho contribuye el desarrollo alcanzado por las denominadas Tecnologías de la Información y la Comunicación (TIC), que juegan un papel muy importante tanto en la creación como en la difusión de la información y que pueden ayudar de forma importante a las personas mayores en su formación formal o informal a lo largo de la vida [3]. De ahí la importancia de conseguir competencias para la alfabetización digital no solamente técnicas sino también pedagógicas dado el enorme potencial de las herramientas digitales [4].

A finales del 2013, la Organización de Naciones Unidas (ONU) informó que para el año 2050 estiman más de dos mil millones de adultos de la tercera edad usarán Internet, lo cual representa un aumento del 300\% [5]. Lo cual implica que esta población hará uso de distintas aplicaciones y dispositivos tecnológicos con mayor frecuencia en las actividades cotidianas que desempeñe. El uso de internet, redes sociales o desde otras aplicaciones permite compartir información entre usuarios, siendo la ubicación o localización una forma de navegar para compartirla en las aplicaciones. Esto requiere aplicaciones más seguras para las personas mayores, ya que son más vulnerables a extraviarse o requerir apoyo para ubicar algún lugar o sitio a donde se desplacen.

Con base a lo anterior, el presente trabajo tiene como propósito el diseño y desarrollo de una Aplicación Móvil para dispositivos con sistema Android con la finalidad de servir como una herramienta de navegación personal para compartir la ubicación de manera rápida y sencilla de los usuarios de la tercera edad con las personas deseadas mediante el uso de un código, almacenado en una base de datos, lo cual brindará mayor seguridad para las personas de la tercera edad. Por otra parte el diseño permite la configuración de interfaces accesibles móviles para las personas de la tercera edad con discapacidad auditiva y/o visual. 
Diseño y desarrollo de una aplicación móvil accesible de navegación individual y localización ...

La aplicación permitirá ubicar al usuario de manera exacta, generar un código único y almacenarlo en la base de datos. Así como la obtención de la información y mostrar la ubicación real del usuario al utilizar Web Services.

El artículo se organiza de la siguiente manera: en la sección 2 presenta la fundamentación teórica y el estado del arte de este trabajo; en la sección 3 se realiza el análisis y diseño de la aplicación móvil; en la sección 4 se presenta la propuesta de la aplicación móvil y la prueba piloto. Finalmente, se presentan las conclusiones y el trabajo futuro en la sección 5 .

\section{Marco teórico}

En esta sección revisaremos los tópicos de las personas de la tercera edad, discapacidad visual y la tecnología. Así como los tipos de aplicaciones móviles, el diseño centrado en el usuario y la experiencia del usuario, siendo relevantes en nuestra propuesta de trabajo.

\subsection{Personas de la tercera edad, discapacidad visual y tecnología}

En general, la tecnología ha tenido que proponer diferentes adaptaciones para poder ser incluyente y accesible a las personas con discapacidades y de la tercera edad. En ese sentido ha surgido la tecnología de apoyo conocida también como "tecnología de adaptación o rehabilitación”. Según Alcantud la define como: "Todos aquellos aparatos, utensilios, herramientas, programas de ordenador o servicios de apoyo que tienen como objetivo incrementar las capacidades de las personas que, por cualquier circunstancia, no alcanzan los niveles medios de ejecución que por su edad y sexo le corresponderían en relación con la población normal" [6]. En la convención de las Naciones Unidas sobre los Derechos de las Personas con Discapacidad, aprobada en 2006 [7], existe la necesidad que se diseñen aplicaciones bajo un diseño universal para todos, pero que no excluye las ayudas técnicas para grupos particulares de personas con distintas discapacidades, lo cual requieren que se adapten a las capacidades específicas, de acuerdo a las normas de accesibilidad, tanto para contenidos, servicios y dispositivos.

\subsection{Aplicaciones móviles accesibles}

Los dispositivos móviles, incluyendo los teléfonos inteligentes y las tabletas, permiten a los usuarios de la tercera edad acceder de forma más sencilla a los servicios de internet a través de las redes móviles e inalámbricas. En el 2012, el mercado mundial móvil se conformó en más de 6500 millones de usuarios, la mayoría de los cuales proceden de países en desarrollo [8] generando la necesidad de desarrollar diversas aplicaciones móviles para distintas generaciones.

Según Gil [9], las aplicaciones móviles conocidas como "Apps", ya se han establecido principios básicos para el diseño de "Apps Accesibles" de acuerdo a la norma "UNE 139802:2009-Requisitos de accesibilidad del software. (ISO 9241- 
171:2008)", los cuales han sido ya adaptados a las necesidades de los dispositivos móviles. Las aplicaciones móviles accesibles han sido definidas como "una aplicación es accesible cuando cualquier usuario, independientemente de su diversidad funcional, puede utilizarla en su dispositivo móvil satisfactoriamente con su sistema de acceso habitual" [9].

Una aplicación móvil se identifica en la actualidad porque puede funcionar en distintos dispositivos móviles como característica principal, sin embargo, dentro de las aplicaciones móviles existen tres tipos [9]:

- Aplicaciones nativas, son aquellas que se desarrollan bajo un lenguaje y un entorno de desarrollo en específico para cada sistema operativo.

- Aplicaciones web, estas son desarrolladas usando leguajes para el desarrollo web como lo son HTML, CSS y JavaScript.

- Aplicaciones Hibridas, como su nombre lo indica tienen un poco de cada tipo de las aplicaciones anteriores.

Las aplicaciones móviles las podemos encontrar de forma gratuita o con un costo en los diferentes sitios como: "AppStore", "GooglePlay" y "Windows PhoneStore", quienes ofrecen servicios de compras en línea, educación, finanzas, navegación, etc., siendo unas de las aplicaciones más utilizadas la geolocalización, la cual ofrece servicios para facilitar las tareas como: mostrar el tráfico, optimización de rutas, lugares de interés social, recordatorios al pasar cierta zona, ver los lugares más visitados, buscar direcciones y otros servicios. Las aplicaciones más usadas en este contexto son:

- Google Maps, es una aplicación móvil de mapeo desarrollado por Google para los sistemas operativos Android e iOS, utiliza la API de Google Maps para su información. Los mapas e información no están incluidos en los mapas de Google instaladas para los dispositivos, se requiere una conexión a Internet para hacer uso de ellos y permite al usuario, descargar la ruta de un punto de referencia a otro, con un área de 10 millas cuadradas $(26 \mathrm{~km} 2)$ alrededor de cualquier punto [10].

- Waze, es la aplicación de tráfico y navegación basada en la comunidad más grande del mundo, dando información de tráfico y ruta en tiempo real por sus usuarios [11].

- Foursquare, consiste en una red social, en la que los usuarios realizan 'check-ins' en los lugares que visitan, comparten recomendaciones y opiniones con sus contactos. Para poder hacerlo de manera efectiva hace uso del GPS del dispositivo [12].

\subsection{Sistemas de posicionamiento}

Los sistemas de posicionamiento, se refieren al método por el cual se puede determinar la posición de un dispositivo, y utilizando servicios adicionales se puede responder preguntas como ¿Dónde estoy?, ¿Qué hay cerca?, ¿Cómo llego allá?, entre otras.

La ubicación puede ser expresada como una descripción textual o en términos espaciales [11]. Una ubicación expresada como un texto es por lo general la dirección 
Diseño y desarrollo de una aplicación móvil accesible de navegación individual y localización ...

de una calle, la ciudad, comuna, código postal, etc. Una ubicación espacial puede ser expresada en términos de coordenadas geográficas usando latitud-longitud-altitud (esta última opcional). La latitud se expresa en grados de 0-90 al norte o sur del ecuador, la longitud en grados de 0-180 al este u oeste del meridiano de Greenwich, y la altitud en metros sobre el nivel del mar. Existen varios métodos [12] para determinar la ubicación de un dispositivo, los cuales varían en las tecnologías empleadas y en la precisión con que entregan el resultado. Siendo las más utilizadas las redes telefónicas, celulares, de área local, Wi-Fi, Bluetooth y GPS entre otros.

- Con respecto al método Cell-ID, es considerado de bajo costo que utiliza la red GSM para determinar la ubicación de un teléfono celular. El identificador de la antena o célula (Cell ID) a la que está conectado el dispositivo es usado para aproximar la ubicación del usuario. La precisión de este método depende del tamaño de la célula: unos 500 metros de exactitud dentro de una ciudad y unos 10 kilómetros en zonas rurales.

- $\quad$ Por otra parte, el método Bluetooth, es una red de corto alcance, este provee un posicionamiento de buena precisión, con máximo de error de unos 10 metros, además de poder entregar posicionamiento vertical. Es muy adecuado para obtener la ubicación dentro de edificios o zonas urbanas pequeñas.

\subsection{El enfoque de la experiencia del usuario}

Para Nielsen y Norman, afirman de la necesidad de distinguir experiencia del usuario y usabilidad, considerando que la usabilidad es un atributo de calidad de una interfaz de usuario asociada a la facilidad de aprender a usar un sistema, su uso eficiente y placentero, mientras que la experiencia del usuario es un concepto más amplio que involucra el análisis de la experiencia de interacción más allá de la relación entre usuario y producto [13].

\section{Metodología de desarrollo de la aplicación móvil}

En esta sección se presenta el análisis, diseño y arquitectura de la aplicación, bajo el enfoque de la metodología ágil y el diseño centrado en el usuario.

\subsection{Metodología del diseño centrado en el usuario y desarrollo de software ágil}

El Diseño Centrado en el Usuario (DCU) es aquel que desde los inicios del desarrollo del producto, hace participar al usuario y lo involucra durante todo el proceso. En este trabajo, se usará una propuesta de DCU Ágil. Esta metodología combina el proceso típico del Diseño Centrado en el Usuario y Desarrollo de Software Ágil (Programación extrema XP, como se muestra en la Figura 1.

En la fase de investigación, necesidades de usuarios y contextos de uso: se ha usado la técnica de investigación contextual (contextual Enquiry) que permite 
observar la motivación del usuario mientras interactúa con un sistema similar al que se va a diseñar. Con respecto a la programación Extrema [14], se aplicó y con la fase de exploración, se determinaron los requerimientos por medio de la creación de Historias de usuarios del grupo focal: "Adultos mayores discapacitados" y se integraron los casos de uso en UML para la aplicación.

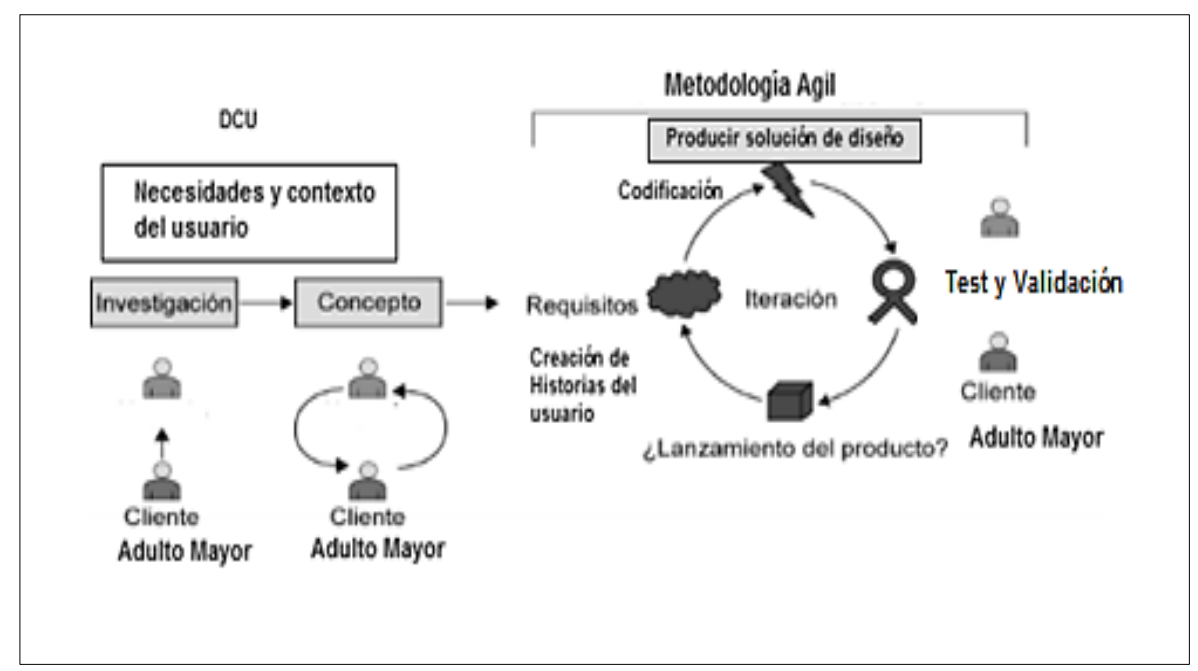

Fig. 1. Metodología de desarrollo de la aplicación móvil.

En la Figura 2, se muestra el usuario "Adulto Mayor" con las actividades a desempeñar y la interacción que tiene con la base de datos del dispositivo y el WebService.
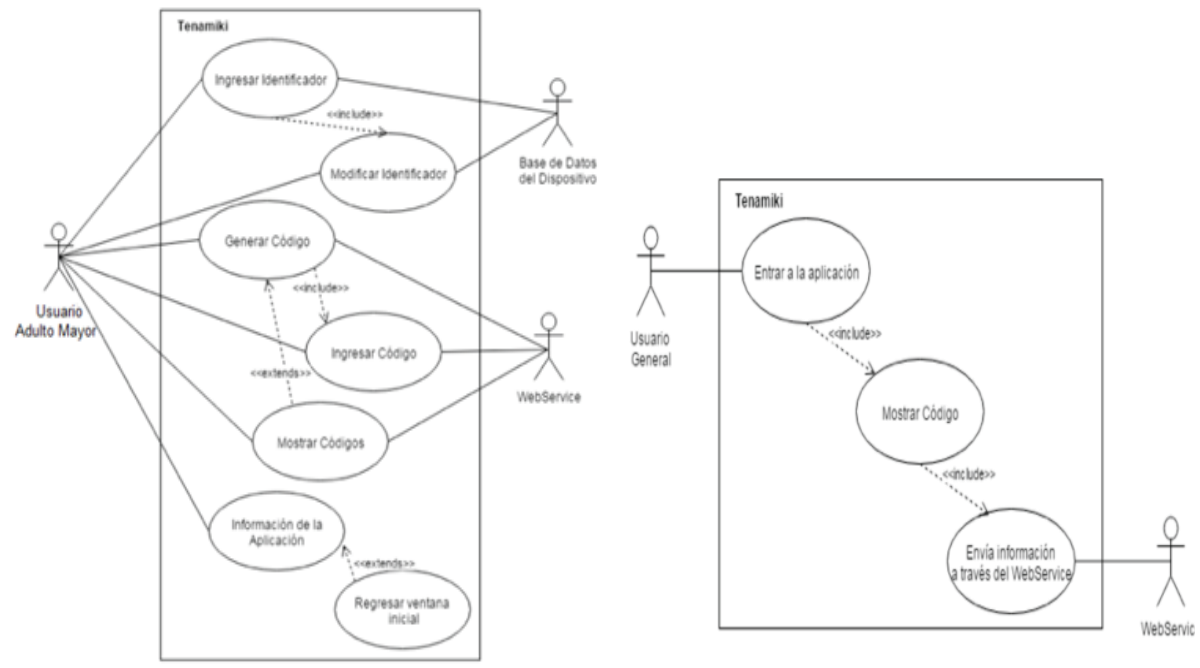

Fig. 2. Casos de uso del sistema. 


\subsection{Arquitectura de la aplicación móvil}

La arquitectura de la aplicación consta de dos partes: la primera parte es la aplicación para dispositivos Android, la cual se conecta al WebService [15], que a su vez se comunica con la base de datos que almacena e intercambia información de la aplicación. Por otra parte la visión de un módulo Web agregado al servidor web con Apache para el portal de aplicaciones de apoyo a adultos mayores de la tercera edad Accesibles (AAMA), como se muestra en la Figura 3.

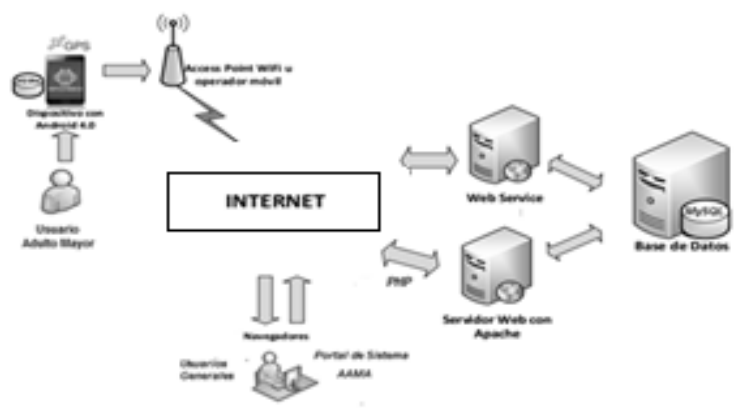

Fig. 3. Esquema de la Arquitectura de la aplicación móvil.

\subsection{Producir prototipo de la aplicación móvil}

El Diseño centrado en el usuario se adoptó para las características propias del usuario de Adulto Mayor con alguna discapacidad visual. Para lo cual se realizó una serie de entrevistas y encuestas con el grupo focal, finalmente se plantearán los prototipos de la aplicación, los cuales se realizaron con el Software Balsamic, como se muestra en la Figura 4.
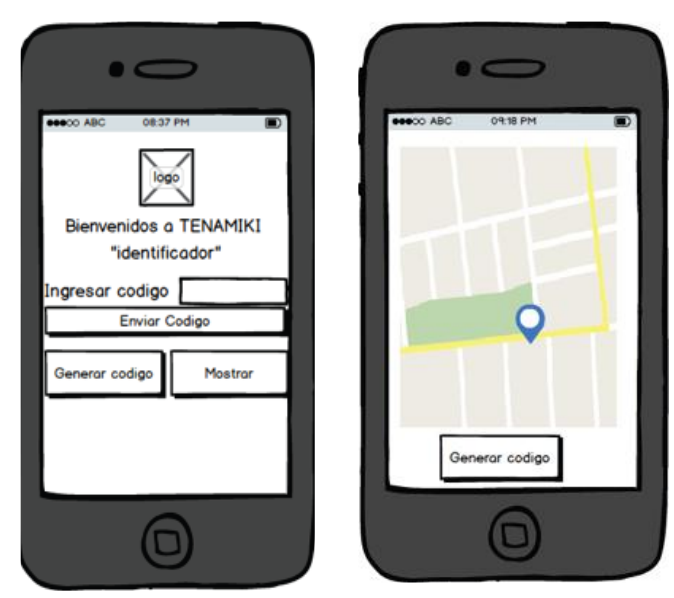

Fig. 4. Esquema de prototipos del la interfaz de la aplicación móvil. 
Los resultados obtenidos fue que la mayoría de los adultos mayores de la tercera edad ya poseen un teléfono inteligente, siendo el sistema operativo que más predomina es Android y de las herramientas que han muy poco utilizado es Google Maps y Waze, pero aseguran que requieren no solo compartir su localización, sino tener certeza de que hayan recibido la localización sus familiares o conocidos y saber cuántos usuarios han consultado la ubicación que han compartido y poder llevar un registro de las personas contactadas por este medio y los datos de la localización de los usuarios.

Por lo cual se planteó las características principales con las que debe contar la aplicación:

- Interface clara e intuitiva: Puesto que lo han de utilizar personas con muy pocas habilidades tecnológicas, se requiere de una interface gráfica muy clara e intuitiva, con pocos botones y que los que haya sean grandes. Además de incluir el modo auditivo de lectura de textos y sonidos.

- Pocas opciones hasta llegar al resultado: Se quiere que la aplicación sea ágil, así que se necesita pocas pantallas y decisiones, para llegar al resultado esperado.

- Práctica: La aplicación debe resultar lo más práctica posible para que el usuario la encuentre de gran utilidad.

\section{Desarrollo y pruebas del prototipo de la aplicación móvil}

En esta sección se muestran los resultados del prototipo de la implementación de la aplicación móvil así como las pruebas de funcionalidad junto con las pruebas de usabilidad obtenidas del grupo focal de usuarios. Las tecnologías utilizadas PHP, MySQL, Webservices, Java, Android Studio 15, Servidor Web: Apache 2.4.10, Emulador Android: Genymotio 2.5.3. Las interfaces finales para la aplicación móvil TENAMIKI (significa en Náhuatl "Encontrase") como se muestran en las Figura 5.
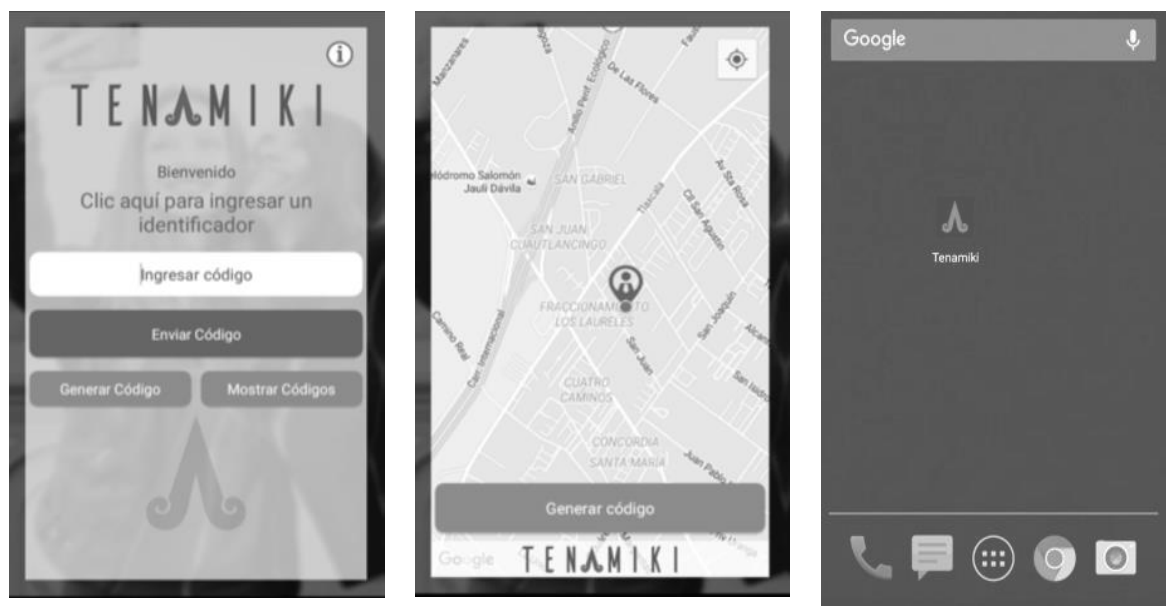

Fig. 5. Pantalla de inicio con elementos visuales para la tercera edad. 
Diseño y desarrollo de una aplicación móvil accesible de navegación individual y localización ...

\subsection{Prueba Piloto y de Usabilidad}

La aplicación móvil se le aplicó una prueba piloto un grupo de 15 usuarios adultos mayores de la tercera edad, de los cuales el $80 \%$ tiene una disminución visual. Para la evaluación de la aplicación, se realizaron pruebas de funcionalidad y usabilidad. Con respecto a las pruebas de Usabilidad presentaron tres posibles escenarios posibles para los usuarios finales, a continuación se describen:

- Situación 1: Al usuario se le entregó la aplicación con la WiFi, GPS y 3G apagados, además se les dio una breve explicación del uso de la aplicación.

- Situación 2: Al usuario se le entregó la aplicación con la WiFi, GPS y 3G prendidos y se le explico el uso de la aplicación.

- Situación 3: Al usuario se le entregó la aplicación con la WiFi, GPS y 3G apagados y no se explicó el uso de la aplicación

Para cada una de las situaciones los usuarios deberán cumplir ciertas tareas para así comprobar el funcionamiento de la aplicación.

- Tarea 1: Ingresar un código generado.

- Tarea 2: Encontrar dónde se muestran los códigos que se han generado.

- Tarea 3: Generar un código en una localización diferente a la que se encuentra actualmente.

- Tarea 4: Cambiar el identificador.

- Tarea 5: Compartir el código.

Después de realizar las pruebas a 15 usuarios 5 por cada situación, se muestran los resultados obtenidos en la Tabla 1 , donde se observa el porcentaje de usuarios que pudieron completar la tarea.

Tabla 1. Resultados Prueba de Usabilidad usando Escenarios.

\begin{tabular}{cccc}
\hline Tareas & Situación 1 & $\begin{array}{c}\text { Escenarios de Test } \\
\text { Situación 2 }\end{array}$ & Situación 3 \\
\hline \hline Tarea no.1 & $90 \%$ & $95 \%$ & $60 \%$ \\
\hline \hline Tarea no.2 & $100 \%$ & $100 \%$ & $100 \%$ \\
\hline \hline Tarea no.3 & $100 \%$ & $100 \%$ & $100 \%$ \\
\hline \hline Tarea no.4 & $100 \%$ & $100 \%$ & $100 \%$ \\
\hline \hline Tarea no.5 & $80 \%$ & $100 \%$ & $80 \%$ \\
\hline Promedio & $94 \%$ & $99 \%$ & $88 \%$ \\
\hline
\end{tabular}

Lo cual refleja que los usuarios con una breve explicación de la situación 1 su desempeño fue del $94 \%$ del cumplimiento de las tareas mientras que los usuarios de la situación 2 al 99\% realizaron las tareas casi en su totalidad y para la situación 3 lograron las tareas en un $88 \%$, esto implica que se presenta una interfaz intuitiva y agradable. Finalmente se aplicó una encuesta de satisfacción de valoración del software [16, 17] la evalúa siete criterios: navegación, interactividad, inmersión, usabilidad, creatividad, efectividad y calidad, con una escala de 1 a 5, cuyo promedio 
obtenido fue de $4.7(94.37 \%)$, a continuación se presentan los resultados en la Figura 6.

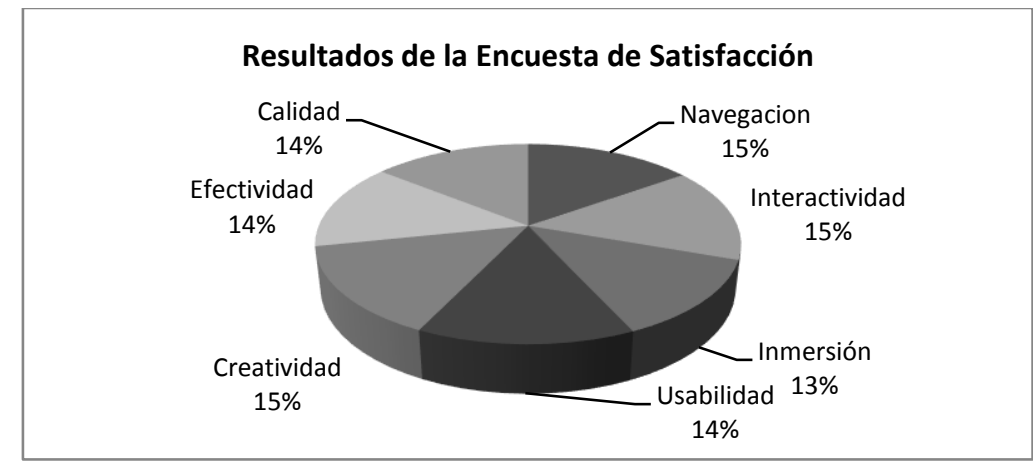

Fig. 6. Resultados de la encuesta de satisfacción de la aplicación móvil.

\section{Conclusiones y trabajo a futuro}

El diseño y desarrollo de una aplicación móvil para dispositivos con sistema Android que permita compartir su ubicación de manera rápida y sencilla a los usuarios de la tercera edad con discapacidad visual, mediante la generación de un código, se implementó alcanzando un porcentaje mayor al 90\% de satisfacción. La Metodología del Diseño Centrado en el Usuario y Desarrollo de Software Ágil, permitió el identificar las características de los adultos mayores de la tercera edad con discapacidad visual en el contexto de la investigación.

Los adultos de la tercera edad se consideran la sociedad cognitiva a la cual se requiere de estudios para integrarlos al uso de las aplicaciones móviles accesible, a través de interfaces amigables para la discapacidad visual y evitar la marginación de personas todavía productivas en su vida, por ello se pretende, brindar un aporte, más allá de las diferencias de capacidades, y se beneficien con las herramientas informáticas como un medio de inserción en un mundo que ofrece cada vez mayores posibilidades de integración en la sociedad.

Las investigaciones que se continúan desarrollando se integrarán en una aplicación para las personas de la tercera edad con alguna discapacidad: auditiva, cognitiva y por supuesto visual; puesto que se requiere contribuir a través de las Tecnologías de la Información y Comunicación para la contextualización social y productividad con calidad de vida.

\section{Referencias}

1. Muñoz, C.: Bienestar Subjetivo y Actividad Social con Sentido Histórico en Adultos Mayores. Revista Hacia la Promoción de la Salud, Disponible en: http://google.redalyc.org/articulo.oa?id=309131077002, pp. 13-26 (2013)

2. Limón, M. R., Ortega, M. C.: Envejecimiento activo y mejora de la calidad de 
Diseño y desarrollo de una aplicación móvil accesible de navegación individual y localización ...

vida en adultos mayores. Revista de Psicología y Educación, 1(6), pp. 225-238 (2011)

3. Trentin, G.: E-learning and the third age. Journal of Computer Assisted Learning, 20(1), 21-30, doi:10.1111/j.1365-2729.2004.00061.x (2004)

4. Moreno, M. D.: Alfabetización digital: el pleno dominio del lápiz y el ratón. Comunicar, 30, 137-146, doi:10.3916/c30-2008-02-007 (2008)

5. Naciones, U.: La sostenibilidad y la inclusión de las personas mayores en el entorno urbano. Disponible en: http://www.un.org/es/events/olderpersonsday (2015)

6. Alcantud, F.: Estudiantes con Discapacidades Integrados en los Estudios Universitarios: Notas para su Orientación. En: Rivas, F. (Ed.), Manual de Asesoramiento y Orientación Vocacional, Primera Edición, Síntesis, Madrid (1995)

7. Naciones, U.: Convención sobre los Derechos de las Personas con Discapacidad. Disponible en: http://www.un.org/esa/socdev/enable/documents/tccconvs.pdf (2015)

8. Johnson, L., Adams, B., Gago, D., Martín, S.: NMC Perspectivas Tecnológicas: Educación Superior en América Latina 2013-2018. Un Análisis Regional del Informe Horizon del NMC, Austin, Texas, The New Media Consortium (2013)

9. Gil, S.: Cómo hacer Apps Accesibles. Centro de Referencia Estatal de Autonomía Personal y Ayuda Técnica, Disponible en: http://www.ceapat.es/ ceapat_01/index.htm, pp. 5-85 (2013)

10. Cuello, J.: Diseñando apps para móviles. Disponible en: http://appdesignbook. com (2005)

11. Fuentes, D., Opitz, I., Oro, N.: Análisis de Aplicación Móvil basada en Geolocalización. Disponible en: http://profesores.elo.utfsm.cl/ agv/elo322/1s16/ projects/reports/Informe_G14_Elo322.pdf (2013)

12. Bernardos, A., Besada, J., Casar, C.: Tecnologías de localización, ETS Ingenieros de Telecomunicación. Universidad Politécnica de Madrid, Disponible en: http://www.upm.es/sfs/Rectorado/Organos\%20de\%20Gobierno/Consejo\%20 Social/Actividades/tecnologias_servicios_para_sociedad_informacion.pdf (2005)

13. Nielsen, J., Norman, D.: The Definition of User Experience. Disponible en: http://www.nngroup.com/articles/definition-user-experience (2014)

14. Extreme Programming. Disponible en: http://www.extremeprogramming.org (2013)

15. Barry, D.: Service Architecture. Disponible en: http://www.service-architecture. com/articles/web-services/soap.html (2016)

16. Acuña, A., Romo, M.: Diseño Instruccional Multimedia. Pearson Education, México (2011)

17. Pino, M. R., Rodríguez, B., Soto, J. G.: Las Personas mayores y las TIC. Un compromiso para reducir la brecha digital. Pedagogía Social, Revista Interuniversitaria, doi:10.7179/PSRI_2015.26.13, 26, pp. 337-359 (2015) 